\title{
TTR
}

Traduction, terminologie, rédaction

\section{Traduction philosophique et signature : le cas de l'indécidable derridien entame en anglais}

\section{René Lemieux}

Volume 32, numéro 2, 2e semestre 2019

La politique des microcentres : la traduction dans des contextes " mineurs " comme transfert culturel complexe

The Politics of Micro-Centers: Translation in "Minor" Contexts as Complex Cultural Transfer

URI : https://id.erudit.org/iderudit/1068909ar

DOI : https://doi.org/10.7202/1068909ar

Aller au sommaire du numéro

Éditeur(s)

Association canadienne de traductologie

ISSN

0835-8443 (imprimé)

1708-2188 (numérique)

Découvrir la revue

Citer cet article

Lemieux, R. (2019). Traduction philosophique et signature : le cas de

l'indécidable derridien entame en anglais. TTR, 32(2), 217-242.

https://doi.org/10.7202/1068909ar

\section{Résumé de l'article}

L'article présente quelques éléments de réception de Jacques Derrida en Amérique à partir de la traduction de ses « mots » indécidables, et particulièrement de celle d'entame. Y est mise à l'épreuve l'hypothèse selon laquelle la réception d'un auteur produira, avec le temps, une homogénéite toujours plus grande des traductions et un nombre toujours plus restreint des traducteurs et des maisons d'édition. Il s'agit d'adapter le principe d'Antoine Berman selon lequel « toute première traduction en appelle à des retraductions " (1995, p. 84) parce qu'elle serait imparfaite, trop idéologique et trop soumise aux normes. Dans le cas de la traduction des sciences humaines et sociales, je pose comme hypothèse qu'on traduit toujours une oeuvre une première fois pour ce qui y est énoncé dans le texte ; c'est la popularité croissante d'un auteur qui augmentera, dans les retraductions, l'intérêt qu'on y porte pour son style, sa langue, etc. Si la plupart des traductions successives de Derrida me permettent d'affirmer cette hypothèse, un exemple la contredit, à savoir la retraduction de La voix et le phénomène par Leonard Lawlor. Quoiqu'elle puisse sembler anodine, la non-conformité de la traduction d'entame par rapport aux traductions habituelles (par broach ou breach, telles que proposées en 1976 par Gayatri Spivak dans Of Grammatology), met au jour un courant mineur de la réception de Derrida aux États-Unis, celui de la phénoménologie, qui ne semble pas catégoriser Derrida comme le fait la réception habituelle. Une étude de ces termes dans la traduction vient donc montrer la force que peut encore avoir un courant de pensée comme la phénoménologie, qui traduit, pourrait-on dire, encore Derrida pour ce qu'il apporte (son objet) plutôt que pour qui il est (son sujet).
Ce document est protégé par la loi sur le droit d'auteur. L'utilisation des services d’Érudit (y compris la reproduction) est assujettie à sa politique d'utilisation que vous pouvez consulter en ligne.

https://apropos.erudit.org/fr/usagers/politique-dutilisation/ 


\title{
Traduction philosophique et signature : le cas de l'indécidable derridien entame en anglais
}

\author{
René Lemieux \\ Université de Sherbrooke
}

\begin{abstract}
Résumé
L'article présente quelques éléments de réception de Jacques Derrida en Amérique à partir de la traduction de ses «mots» indécidables, et particulièrement de celle d'entame. Y est mise à l'épreuve l'hypothèse selon laquelle la réception d'un auteur produira, avec le temps, une homogénéité toujours plus grande des traductions et un nombre toujours plus restreint des traducteurs et des maisons d'édition. Il s'agit d'adapter le principe d'Antoine Berman selon lequel «toute première traduction en appelle à des retraductions» $(1995$, p. 84) parce qu'elle serait imparfaite, trop idéologique et trop soumise aux normes. Dans le cas de la traduction des sciences humaines et sociales, je pose comme hypothèse qu'on traduit toujours une œuvre une première fois pour ce qui y est énoncé dans le texte; c'est la popularité croissante d'un auteur qui augmentera, dans les retraductions, l'intérêt qu'on y porte pour son style, sa langue, etc. Si la plupart des traductions successives de Derrida me permettent d'affirmer cette hypothèse, un exemple la contredit, à savoir la retraduction de La voix et le phénomène par Leonard Lawlor. Quoiqu'elle puisse sembler anodine, la nonconformité de la traduction d'entame par rapport aux traductions habituelles (par broach ou breach, telles que proposées en 1976 par Gayatri Spivak dans Of Grammatology), met au jour un courant mineur de la réception de Derrida aux États-Unis, celui de la phénoménologie, qui ne semble pas catégoriser Derrida comme le fait la réception habituelle. Une étude de ces termes dans la traduction vient donc montrer la force que peut encore avoir un courant de pensée comme la phénoménologie, qui traduit, pourrait-on dire, encore Derrida pour ce qu'il apporte (son objet) plutôt que pour qui il est (son sujet).

Mots-clés : déconstruction, Jacques Derrida, études de la réception, histoire des idées, traduction des sciences humaines et sociales
\end{abstract}

\section{Abstract}

The article presents some elements of Jacques Derrida's reception in the United States, based on the translation of his undecidable "words", especially on the translation of the French term entame. It tests the hypothesis that the 
reception of an author will produce, over time, an ever-greater uniformity of translations and an ever-smaller number of translators and publishers. The purpose of the article is to adapt Antoine Berman's principle according to which any first translation calls for retranslations (1995, p. 84), because it is imperfect, too ideological and overly subjected to norms. Regarding the field of translation in humanities and social sciences, I make the assumption that the first time a work is translated, it is always for its content; it is the growing popularity of the author that will increase, in retranslations, the interest in his style, his way of playing with the language, etc. Most successive translations of Derrida allow me to confirm this assumption, but one example stands apart: Leonard Lawlor's retranslation of La voix et le phénomène. However trivial it may seem, this exception among the usual renderings of entame (by broach and/or breach as proposed in 1976 by Gayatri Spivak in Of Grammatology), reveals a minor stream in Derrida's reception in the United States-namely phenomenological theory - which does not seem to categorize him the way the major part of the reception community does. Therefore, a study of these terms in translation shows the influence still held by a school of thought like phenomenological theory that, arguably, still translates Derrida for what he offers (the object of the text), rather than who he is (the subject of the text).

Keywords: deconstruction, Jacques Derrida, reception studies, history of ideas, translation in humanities and social sciences

\section{Introduction : de la réception d'un auteur à l'étude de sa traduction}

L'objectif de cet article est de présenter quelques éléments de réception de Jacques Derrida en Amérique, principalement à partir de la traduction de ses «indécidables», ces mots auxquels on ne peut pas attribuer qu'une seule et unique signification. Je souhaite y mettre à l'épreuve l'hypothèse selon laquelle la réception d'un auteur produira, avec le temps, une homogénéité toujours plus grande des traductions et un nombre toujours plus restreint des traducteurs et des maisons d'édition. Il s'agit d'adapter le principe d'Antoine Berman selon lequel «toute première traduction en appelle à des retraductions" (1995, p. 84) parce qu'elle serait imparfaite, trop idéologique et trop soumise aux normes. Dans le cas de la traduction en sciences humaines et sociales, je pose comme hypothèse qu'on traduit toujours une œuvre une première fois pour ce qui y est énoncé dans le texte (ce que je nomme son "quoi») alors que la popularité croissante d'un auteur augmente l'intérêt qu'on y porte pour son style, sa langue, etc. (on traduirait alors son «qui») (Lemieux, 2015b). Si la plupart des traductions successives de Derrida permettent d'appuyer cette hypothèse, un exemple la contredit, à savoir la retraduction de La voix et le phénomène ${ }^{1}$ par Leonard Lawlor (Voice and Phenomenon, 2010), après

1. Il s'agit d'un commentaire d'Edmund Husserl par Derrida. 
une première traduction, Speech and Phenomena, par David Allison en 1973. Ainsi, je présenterai d'abord les éléments théoriques nécessaires à la compréhension du concept de «retraduction» (notamment avec Berman), puis j'aborderai le cas du terme entame, qui permet justement de montrer que, derrière des choix de traduction, il y a toute une histoire de la réception qui se joue.

\section{La retraduction chez Antoine Berman}

Berman formule une thèse des plus intéressantes pour expliquer le processus de retraduction. Pour lui, les retraductions qui suivent la première traduction sont faites pour l'auteur, mais contre les anciennes traductions. Cela est possible grâce à sa définition de la retraduction, qui n'est pas simplement le fait de traduire un texte (ou un auteur) déjà traduit. Pour Berman, en effet, la retraduction se présente comme un «espace» créé par la relation dynamique entre trois éléments :

Celui qui re-traduit n'a plus affaire à un seul texte, l'original, mais à deux, ou plus, ce qui dessine un espace spécifique :

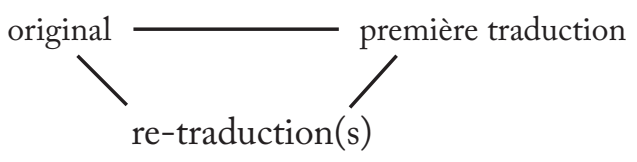

[...] Tout se passe comme si la secondarité du traduire se redoublait avec la re-traduction, la «seconde traduction" (d'une certaine manière, il n'y en a jamais une troisième, mais d'autres «secondes»). Je veux dire par là que la grande traduction est doublement seconde : par rapport à l'original, par rapport à la première traduction. (Berman, 1999, p. 105)

Pour Berman, la première traduction est toujours «adaptatrice» ou "parodique»; elle s'intéresse d'abord au public. Dans un numéro de la revue Palimpsestes consacré à la retraduction, le directeur de la revue, Paul Bensimon, écrivait :

La première traduction procède souvent - a souvent procédé - à une naturalisation de l'œuvre étrangère; elle tend à réduire l'altérité de cette œuvre afin de mieux l'intégrer à une culture autre. Elle s'apparente fréquemment - s'est fréquemment apparentée - à l'adaptation en ce quelle est peu respectueuse des formes textuelles de l'original. La première traduction vise généralement à acclimater l'œuvre étrangère en la soumettant à des impératifs socio-culturels qui privilégient le destinataire de l'œuvre traduite. (1990, p. ix) 
Ainsi, ce sont les traductions subséquentes qui pourront s'arracher des contraintes de la culture cible et alors être plus littérales, et, par cela même, se rapprocher de l'original.

Si les retraductions sont nécessaires, c'est parce que, contrairement à l'original qui reste jeune, les traductions ont tendance à vieillir; elles sont soumises au temps. Les traductions de la Lettre qui intéressent Berman, elles, sont pourtant des traductions qui ne vieillissent pas : elles "perdurent à l'égal des originaux et [...], parfois, gardent plus d'éclat que ceux-ci» (Berman, 1990, p. 2). Si la traduction comme produit peut "vieillir», contrairement à un original, c'est parce qu'elle se constitue dans une défaillance, un caractère toujours plus grand dans les premières traductions :

Tout se passe comme si les forces anti-traductives qui provoquent la «défaillance» étaient, ici, toutes puissantes. Si la défaillance, c'est-à-dire simultanément l'incapacité de traduire et la résistance au traduire, affecte tout acte de traduction, il y a néanmoins une temporalité de cet acte (temporalité aussi bien psychologique que culturelle et linguistique) qui fait que c'est en son début (dans la première traduction) que la défaillance est à son comble. La retraduction surgit de la nécessité non certes de supprimer, mais au moins de réduire la défaillance originelle. (ibid., p. 5)

Il est possible de retraduire grâce à des «moments favorables» (ou kairos), "[a]près maintes introductions érudites, scolaires, maintes adaptations" (ibid., p. 6), comme si tout un travail en sous-main était nécessaire pour en arriver à une traduction plus près de la perfection. Or, apprécier le perfectionnement de la traduction dans le temps comme le fait Berman, c'est-à-dire juger une "première» traduction comme «défaillante», nécessite de concevoir le jugement sur la traduction comme un jugement dernier. Ce n'est qu'après coup, lorsqu'est apparue la «véritable» traduction, qu'un tel jugement est possible. Le jugement bermanien sur les "grandes retraductions" n'est pensable qu'à partir d'un certain regard que l'on porte sur l'œuvre a posteriori.

Cette théorie de la retraduction mérite qu'on s'y repenche à partir de l'histoire de la réception d'une œuvre. Dans le domaine des sciences humaines et sociales, la retraduction se présente à première vue comme un exercice très différent de la traduction littéraire qu'avait en tête Berman. On verra toutefois que les conséquences (une plus grande attention à la Lettre, par exemple) sont souvent les mêmes. Les sections qui suivent viseront à mettre à l'épreuve la théorie bermanienne avec un exemple de traduction contemporain. 


\section{La traduction des concepts en sciences humaines et sociales}

Traduire les sciences humaines et sociales signifie s'intéresser aux «concepts» ${ }^{2}$. Comme tout discours spécialisé, les sciences humaines et sociales commanderaient un souci particulier pour les termes reconnus dans la communauté de locuteurs concernée. La philosophie pousserait plus loin cette logique en faisant de ses termes des expressions ou mots particuliers à certains auteurs et à leurs lecteurs. C'est ainsi que le propose Siobhan Brownlie :

Dans un texte philosophique, la terminologie technique revêt une très grande importance qualitative, même si, quantitativement, la langue générale est prépondérante. Puisque les termes philosophiques sont élaborés par des individus dans un contexte culturel particulier et dans une langue spécifique, il n'en existe pas, par contraste avec les termes technologiques et scientifiques, d'équivalents préexistants dans une autre langue, du fait de l'élaboration simultanée de concepts et d'activités dans le domaine en question. Ce sont les traducteurs du philosophe qui ont donc la responsabilité de créer des équivalents. (2002, p. 297)

La proposition de Brownlie, comme celle de Berman présentée plus haut, semble anhistorique, à la fois pour ce qui est de l'histoire de la philosophie en général (on ne traduit pas la philosophie aujourd'hui de la même manière qu'autrefois ${ }^{3}$ ) et de la traduction dans la réception d'un auteur particulier. Jacques Derrida devient un cas particulier, car ses «concepts», qu'on peut appeler avec lui des «indécidables» (il utilise également l'expression «quasi concept» vers la fin des années 1980), visent toujours à jouer avec la possibilité même de déterminer une signification ultime à un terme. Le plus connu est sans doute le terme différance, dont l'exemplarité permet de prendre la mesure de la difficulté pour le traducteur. Aux États-Unis, le terme n'a jamais été traduit. Son intraduisibilité serait la conséquence d'abord de l'imperceptibilité auditive de sa différence avec différence, ensuite, entre autres,

2. Pour une explication plus générale de cette affirmation, on pourra consulter Heim et Tymowski (2006). Pour des exemples de «mauvaises» traductions dans ce domaine, on pourra consulter Wallerstein (1981).

3. Pour un exemple d'analyse des traductions philosophiques, tant dans la perspective diachronique de l'histoire de la réception que celle, synchronique, des différences nationales, on pourra consulter Lemieux (2009) sur les traductions anglaises, portugaises et finnoises de l'indécidable derridien différance.

4. Pour ce qui est de la retraduction d'un auteur, un des exemples les plus connus est celui du terme Dasein de Martin Heidegger, qui sera tour à tour traduit par réalité bumaine, être-là, être-le-Là (suggestion de Heidegger lui-même) et gardera finalement sa forme originale avec Dasein. Voir notamment Cassin (2004). 
de son indécidabilité sémantique entre une signification spatiale (différencier, en anglais : to differ) et temporelle (différer dans le temps, en anglais : to defer). La réception de ce terme est toutefois différente ailleurs, notamment en portugais, où il a été rendu par une douzaine de termes (Lemieux, 2009). Ainsi, lorsqu'on compare les traductions d'un même terme dans des langues différentes, on peut prendre la mesure des difficultés que pose la traduction philosophique. Or, pour comprendre l'évolution interne de la réception d'une philosophie, il faut employer une méthodologie différente, qui s'intéresse aux conditions d'exercice de la traduction et, en particulier, à l'origine disciplinaire des traducteurs. D'où viennent les traducteurs? Quelles sont leurs spécialités? Quel public visent leurs traductions?

\section{Une approche sociale du retour en traduction}

Quelle est l'importance de la spécialisation en traduction philosophique? Cette spécialisation, comme je tenterai de le montrer, a plus à voir avec son rapport à la communauté des lecteurs (la réception de l'auteur) qu'aux compétences en traduction de la personne choisie pour faire le travail. Le mythe de Babel pourrait étonnamment nous fournir un indice d'une potentielle nouvelle approche sociale de la traduction des sciences humaines et sociales ${ }^{5}$. Rappelons que dans la Genèse (chapitre 11, versets 1 à 9) il est raconté que le peuple rassemblé, parlant une même langue, a voulu se construire une tour allant jusqu'aux cieux. Ayant vu cela, Dieu est descendu et a détruit la tour, multipliant les langues et dispersant l'humanité. Ce serait une leçon de modestie : si le peuple était parvenu à construire la tour, il aurait été capable de tout accomplir.

Jacques Derrida parle de ce mythe dans le texte «Des tours de Babel» (1987), où il cite la première version de la traduction de la Bible par André Chouraqui (1985). L'objectif de Chouraqui était de traduire de manière hyperlittérale (langues, par exemple, devenant lèrores puisqu'en hébreu, la métaphore pour la langue est lèvre). Le projet de Chouraqui achoppe au moment où il doit traduire le mot Babel, mot incertain, indécidable, qui est à la fois nom propre (Babel est le nom de la tour) et nom commun (signifiant «confusion» ou «brouillage»). Entre ces deux options, Chouraqui refuse de choisir et traduit «Babel, confusion» (Derrida, 1987, p. 206-207). Cette indétermination est possible en hébreu parce que la distinction entre le nom propre et

5. Pour une explication plus longue de cet usage du mythe de la tour de Babel, on pourra consulter Lemieux (2015a). 
le nom commun est au cœur de la grammaire hébraïque. En effet, comme l'indique Spinoza dans sa grammaire hébraïque, le nom est la base de cette langue :

On divise, en latin, le discours en huit parties; mais on peut douter qu'il en soit de même en hébreu. En effet - si l'on excepte les interjections, les conjonctions et une ou deux particules - tous les mots hébreux ont la valeur et les propriétés du nom. Et c'est parce que les grammairiens ne l'ont pas compris qu'ils ont cru irrégulières beaucoup de choses parfaitement régulières - si l'on se réfere à l'usage de la langue - et qu'ils ont ignoré un certain nombre de choses nécessaires pour connaitre et parler la langue hébraïque. (2006, p. 65)

Si on imagine mal, dans des langues flexionnelles comme le français ou l'anglais, qu'une préposition puisse se comprendre comme un pluriel, c'est tout simplement parce que ces langues ne fonctionnent pas selon la même logique. En hébreu, nous dit Spinoza, tout découle du «nom », y compris les verbes, dont les infinitifs se modifient comme des noms, les futurs se dérivent de l'infinitif à l'état de régime, et les passés se présentent sous des formes adjectivales. Or, explique Joël Askénazi, l'un des traducteurs de la grammaire de Spinoza, le nom en lui-même peut être distingué :

La seule distinction grammaticalement fondée à l'intérieur du discours hébraïque est donc la distinction entre le nom commun et le nom propre: le nom propre n'exige pour être compris aucune détermination (qu'elle soit génitivale ou indicative), il ne subit aucune modification de consonne ou de voyelle. (Askénazi, dans Spinoza, 2006, p. 19)

On peut donc en comprendre que tout nom commun hébreu pourrait éventuellement prendre la valeur de nom propre. C'est bien là le problème de l'indécidabilité de Babel-confusion, qui peut à la fois se voir au sens propre et au sens figuré. Le verset 4 du mythe de Babel nous donne toutefois un indice encore plus important. On peut y lire (dans la version de Lemaistre de Sacy, 1759) : "Ils s'entredirent encore : Venez, faisons-nous une ville et une tour qui soit élevée jusqu'au ciel; et rendons notre nom célèbre avant que nous nous dispersions par toute la terre.» Ce «nom », en hébreu, se dit shem (ou sem, שָׁ) et signifie, bien entendu, à la fois «nom propre» et "nom commun ", comme il est aussi le nom propre de Shem, un des fils de Noé. L'interdit exprimé par le mythe peut dès lors signifier celui de «se donner un nom », une « renommée » ou une « réputation» (dans la traduction de Calvin de 1560). 
On peut faire de cet interdit une manière d'articuler le rapport entre ce qu'on pourrait appeler, d'une part, le « quoi » de l'œuvre au sens où l'œuvre dirait quelque chose, c'est-à-dire son objet ou son énoncé, et, d'autre part, le « qui » de l'œuvre, à savoir son sujet, l'énonciateur. La question à se poser en étudiant une traduction serait donc la suivante : $\mathrm{a}$ - $\mathrm{t}$-on traduit quelque chose ou a-t-on traduit quelqu'un? Cette manière de formuler la question n'est pas sans rappeler la typologie proposée par Katharina Reiß (1976) : la traduction de la philosophie se situerait entre le texte informatif (sachorientiert, centré sur l'objet ou le référent) et le texte expressif, centré sur l'émetteur (senderorientiert). Reprenant cette distinction, Jean-René Ladmiral qualifie le texte philosophique d'informatif et d'expressif, et considère que sa traduction devrait prendre en compte à la fois l'objet ou le référent et l'émetteur (1981, p. 23). Or, contrairement à cette théorisation typique du skopos, ce qu'on doit essayer de penser à partir de la question dédoublée - traduire quelque chose ou traduire quelqu'un -, c'est moins le type de texte du point de vue de celui qui l'écrit, où son destinataire serait prédéterminé et essentiellement toujours le même, mais bien du point de vue de celui qui le reçoit. Cette destinée du texte, sa réception, n'est pas nécessairement décidée d'avance.

En analysant la traduction dans la réception d'un auteur, on remarque qu'avec la retraduction se produit une transformation du quoi au qui : au départ, on traduit le «quelque chose» de l'œuvre; on traduit l'œuvre parce qu'elle apporte quelque chose de nouveau, par exemple, à une discipline. La retraduction implique que ce n'est plus ce que dit l'auteur qui importe, mais qui il est. Lorsque l'auteur est retraduit, on met l'accent sur son style, ses formules; on accorde plus d'importance à ses nouveautés terminologiques, à ses créations conceptuelles. Ce ne sont donc pas seulement les bons auteurs qui sont retraduits. Cela veut aussi dire que la retraduction fabrique l'image d'un auteur, avec ses concepts particuliers, ses figures de style, etc. L'immortalité de l'œuvre passe par le «qui» ou ce qu'on pourrait appeler la signature (Lemieux, 2015b, p. 130-206), c'est-à-dire un agencement d'auctorialité, de pratique de la langue et de réseau conceptuel. Ce qui compte pour le traducteur, lorsque l'auteur est suffisamment reconnu comme tel, c'est la fidélité à la signature, et la traduction devient rapidement une contresignature à cette signature, une deuxième signature qui authentifie la première. 


\section{Exemple ou contre-exemple : entame et les limites méthodologiques}

Cette hypothèse du passage du quoi au qui dans la réception d'un auteur fonctionne très bien avec la traduction portugaise de Derrida mentionnée plus haut. Les premières traductions de Derrida dans les années 1970 étaient plus libres; ce qui intéressait les premiers agents de la réception, c'était ce qu'apportait Derrida en sémiologie, discipline alors très en vogue. À partir des années 1990, fort probablement grâce à sa renommée aux États-Unis, on traduit Derrida pour luimême, avec plus de littéralité et, éventuellement, des formes nouvelles d'intraduisibilité (Lemieux, 2009).

Un indécidable derridien se montre toutefois particulièrement difficile à analyser: entame. Ce terme peut servir d'indicateur pour valider mon hypothèse, car il n'a jamais été thématisé par la réception derridienne (en particulier aux États-Unis). Il s'agit d'un indécidable somme toute mineur dans l'œuvre de Derrida; on le trouve d'ailleurs assez rarement chez les commentateurs de Derrida 6 . On rencontre le terme lui-même dès 1953-1954 dans Le problème de la genèse dans la philosophie de Husserl, mémoire rédigé par Derrida sous la direction de Maurice de Gandillac pour l'obtention du diplôme d'études supérieures (publié en 1990). Le concept d'entame est présent dans les premiers livres de Derrida ${ }^{7}$; il s'y manifeste comme nom commun, comme verbe ou comme adjectif (généralement sous la forme négative inentamé), et il est défini (ou pris dans une chaîne d'indécidable) dans les livres de 1972. Ce n'est que plus tard que Derrida, de manière métadiscursive, relèvera le terme entame dans un entretien avec JeanLouis Houdebine et Guy Scarpetta, à l'intérieur d'une réflexion plus générale sur l'usage des indécidables et la logique du ni-ni qui les sous-tend :

[...] le pharmakon n'est ni le remède, ni le poison, ni le bien ni le mal, ni le dedans ni le dehors, ni la parole ni l'écriture; le supplément n'est ni un plus ni un moins, ni un dehors ni le complément d'un dedans, ni un accident, ni une essence, etc.; l'bymen n'est ni la confusion ni la distinction, ni l'identité ni la différence, ni la consommation ni la virginité, ni le voile

6. Le terme est absent du Vocabulaire de Jacques Derrida de Charles Ramond (2001). Le Derrida Dictionary de Niall Lucy (2004) ne l'inclut pas non plus, pas plus que le Glossário de Derrida, premier glossaire en portugais par Silviano Santiago (1976).

7. À 40 reprises dans De la grammatologie (1967), à 11 reprises dans La voix et le phénomène (1967), à 21 reprises dans L'écriture et la différence (1967), à 3 reprises dans Marges - de la philosophie (1972), à 7 reprises dans La dissémination (1972) et à 7 reprises dans Positions (1972), dont dans une question posée. 
ni le dévoilement, ni le dedans ni le dehors, etc.; le gramme n'est ni un signifiant ni un signifié, ni un signe ni une chose, ni une présence ni une absence, ni une position ni une négation, etc.; l'espacement, ce n'est ni l'espace ni le temps; l'entame, ce n'est ni l'intégrité (entamée) d'un commencement ou d'une coupure simple ni la simple secondarité. $\mathrm{Ni} /$ ni, c'est à la fois ou bien ou bien; la marque est aussi la limite marginale, la marche, etc. (Derrida, 1972c, p. 58-59)

Il n'est pas inutile de donner quelques explications sémantiques et étymologiques sur le terme pour comprendre son usage chez Derrida. Selon le Dictionnaire historique de la langue française (Rey, 2006), le verbe entamer (XII ${ }^{e}$ siècle) serait issu du bas latin intaminare, " toucher à", proprement "souiller», "profaner», et signifie d'abord "toucher (quelque chose d'intact) en en retirant une partie», puis "couper (une partie du corps) en incisant». Toujours selon ce dictionnaire, le mot a pris avec le temps différents sens: au propre tant sexuel ("entamer une femme», c'est la déflorer) que militaire ("entamer le front», c'est lui porter atteinte), et au figuré («entamer un discours», c'est le commencer; "entamer des provisions", c'est les diminuer en leur retranchant une partie). Si certains ont pu voir dans ce verbe un dérivé

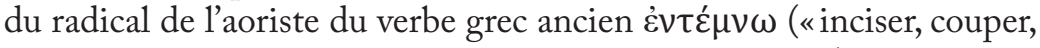
immoler" au sens de "couper une victime, la sacrifier»), ce n'est pas l'avis d'Émile Littré :

[...] du latin attaminare, mettre la main sur quelque chose, prendre.

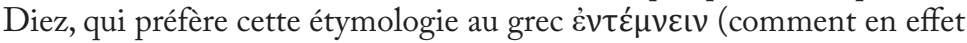
ce mot grec serait-il entré dans les langues romanes?), et au celtique tam, mordre, remarque que les langues romanes changent non rarement le préfixe d'un mot latin : ainsi dans con-vier, venant de in-vitare, cum, au lieu de in. Le celtique (bas-breton, tam; écossais, teum, irl. teuman) conviendrait plus directement au sens; mais les formes entamenar $\mathrm{du}$ provençal, entanpner du français, semblent indiquer le latin. On remarquera endamer de J. de Condet qui était du Hainaut, et les patois voisins du Hainaut qui ont aussi le $d$. Ce $d$ ne peut guère s'expliquer que par une confusion avec dam, dommage. (1874, p. 1418)

Si donc on peut dire que le verbe entamer se rapporte au toucher (d'intaminare ou d'attaminare) dans la mesure où il signifierait originellement un sujet qui toucherait un objet, il a pris de nouveaux sens, y compris son corollaire, puisque le sujet entamant est lui aussi entamé par son action. Ce dédoublement du sens est présent chez Derrida, qui utilisait souvent cette indétermination. Dans le cas des traducteurs et traductrices de Derrida, c'est Gayatri Spivak la première, en 1976, qui 
notera le terme dans sa préface à Of Grammatology. Elle y fait trois mentions d'entame. Dans la première, elle rappelle des indécidables comme trace, différance, dissémination et hymen, puis affirme :

As such it reflects the structure of differance, as does a holding in "reserve", and the "entame"-both beginning something and breaking into something, both origin and trace. (Spivak, 1976, p. 1xxi)

La deuxième occurrence est une citation provenant de la première traduction de Positions qu'elle reprend:

[...] the entame is neither the [marred] integrity of a beginning or $<$ of $>$ a simple cut nor simply the secondary state. (Spivak, 1976, p. 1xxii) ${ }^{8}$

La dernière référence à entame se présente lorsque Spivak décrit les difficultés de traduction qu'elle a rencontrées :

My special worry is "entamer". As we have seen, it is an important word in Derrida's vocabulary. It means both to break into and to begin. I have made do with "broach" or "breach", with the somewhat fanciful confidence that the shadow-word "breach" or "broach" will declare itself through it. With "entamer" as well as with other words and expressions, I have included the original in parenthesis whenever the wording and syntax of the French seemed to carry a special charge. To an extent, this particular problem informs the entire text. (1976, p. lxxxv-lxxxvi)

L'usage des parenthèses mentionné par Spivak ne s'observe qu'une fois dans le cas d'entame, à sa dernière occurrence dans Of Grammatoloty, où il rendu par breaches (Derrida, 1976b, p. 306). De plus, malgré ce qu'en dit Spivak, l'indécidable entame n'a pas été traduit uniquement par broach ou breach. En effet, les 39 occurrences (sans compter la seule qui se trouve dans une citation de Rousseau) sont rendues d'une dizaine de façons différentes. Celles-ci sont présentées dans le tableau 1 (page suivante). On pourra en outre consulter l'Annexe A pour voir l'ensemble des occurrences en contexte, telles qu'elles se présentent dans De la grammatologie.

8. Spivak cite la version (sans mention du traducteur ou de la tra ductrice) publiée dans Diacritics (Derrida, 1972d), qu'elle corrige très légèrement (l'ajout de of que j'ai encadré dans l'extrait cité par des chevrons simples). Cette version, il faut le noter, ne traduit aucun des mots présentés comme indécidables, mais les transcrit tels quels. 
Tableau 1. Traduction d'entame dans Of Grammatoloty

\begin{tabular}{|c|c|c|}
\hline & Information grammaticale & Rendus (nbre d'occurrences) \\
\hline & \multicolumn{2}{|l|}{ Fonction verbale } \\
\hline $\begin{array}{l}\left(s^{\prime}\right) \text { entamer } \\
(3)^{9}\end{array}$ & $\begin{array}{l}\text { infinitif (y compris forme } \\
\text { réfléchie) }\end{array}$ & $\begin{array}{l}\text { broaching (2), violated itself } \\
\text { (1) }\end{array}$ \\
\hline entame (13) & indicatif présent & $\begin{array}{l}\text { broaches }(6), \text { breaches }(2) \text {, } \\
\text { cuts }(2), \text { ushered (1), begins } \\
(1), \text { breaks in (1) }\end{array}$ \\
\hline $\begin{array}{l}\text { n'entame/nt } \\
\text { pas (2) }\end{array}$ & $\begin{array}{l}\text { indicatif présent, avec } \\
\text { négation }\end{array}$ & does/do not interfere (2) \\
\hline s'entame (4) & $\begin{array}{l}\text { indicatif présent, forme } \\
\text { réfléchie }\end{array}$ & $\begin{array}{l}\text { is broached ( } 3) \text {, is broached } \\
\text { from itself (1) }\end{array}$ \\
\hline (s')entamait (2) & $\begin{array}{l}\text { indicatif imparfait (y compris } \\
\text { forme réfléchie) }\end{array}$ & $\begin{array}{l}\text { breached (1), broached itself } \\
\text { (1) }\end{array}$ \\
\hline a entamé (2) & $\begin{array}{l}\text { indicatif passé composé, } \\
\text { introduit par l'auxiliaire avoir }\end{array}$ & $\begin{array}{l}\text { has infiltrated (1), has } \\
\text { broached (1) }\end{array}$ \\
\hline $\begin{array}{l}\text { n'ait pas } \\
\text { entamé (1) }\end{array}$ & $\begin{array}{l}\text { subjonctif passé, introduit } \\
\text { par l'auxiliaire avoir, forme } \\
\text { négative }\end{array}$ & has not broached (1) \\
\hline $\begin{array}{l}\text { (en) entamant } \\
\text { (2) }\end{array}$ & $\begin{array}{l}\text { participe présent (y compris } \\
\text { gérondif) }\end{array}$ & breaching (2) \\
\hline \multirow[t]{2}{*}{ entamerait (1) } & conditionnel, forme réfléchie & would infiltrate (1) \\
\hline & \multicolumn{2}{|l|}{ Fonction nominale } \\
\hline \multirow[t]{2}{*}{ entame (1) } & nom commun & broaching (1) \\
\hline & \multicolumn{2}{|l|}{ Fonction adjectivale } \\
\hline $\begin{array}{l}\text { (laisser/être) } \\
\text { entamée (2) }\end{array}$ & $\begin{array}{l}\text { participe passé, forme } \\
\text { positive }\end{array}$ & $\begin{array}{l}\text { leaving breached (1), be } \\
\text { broached (1) }\end{array}$ \\
\hline $\begin{array}{l}\text { (aucune n'a } \\
\text { été/ne soient } \\
\text { pas) entamées } \\
\text { (2) }\end{array}$ & $\begin{array}{l}\text { participe passé, forme } \\
\text { négative }\end{array}$ & $\begin{array}{l}\text { no ... has been breached ( } 1) \text {, } \\
\text { are not broached (1) }\end{array}$ \\
\hline inentamé (4) & $\begin{array}{l}\text { adjectif formé à partir } \\
\text { d'inentamer }\end{array}$ & intact (3), unblemished (1) \\
\hline
\end{tabular}

9. Le contenu des parenthèses correspond au nombre d'occurrences. 
Si on fait abstraction des emplois de l'adjectif inentamé, on constate que Spivak utilise 9 verbes différents pour traduire les 35 occurrences d'entame : broach (18 occurrences sur 35 , ou $\approx 51 \%$ ), breach (7 occurrences, ou $20 \%$ ), cut (2), interfere (2), infiltrate (2), violate (1), usher (1), begin (1) et break in (1). Dans l'original, on trouve cinq fois le verbe dans sa forme négative, traduit par broach et interfere à deux reprises, et par breach à une reprise. Le verbe dans sa forme réflexive, employé six fois dans l'original, a été généralement rendu par broach (5 occurrences), puis par violate (1) (l'usage de itself s'observe dans trois cas). On pourrait continuer ce dénombrement, en distinguant le verbe selon sa temporalité ou son aspectualité, en fonction de l'usage d'auxiliaires ou de pronoms personnels, etc. Notons simplement l'exceptionnalité de l'adjectif inentamé, qui pourrait être pensé en relation avec entame, mais que Spivak traduit principalement par intact et à une reprise par unblemished. Il y aurait peut-être eu un moyen de montrer le doublet broach/breach dans le vocable derridien, lorsque Derrida utilise le concept d'entame sous deux formes différentes dans une même phrase, ce qui survient à deux reprises dans De la grammatologie :

Elle a commencé par entamer l'aliénation et elle finit par laisser entamée la réappropriation. (Derrida, 1967a, p. 206)

Differance began by broaching alienation and it ends by leaving reappropriation breached. (Derrida, 1976b, p. 143)

Une vie qui n'ait pas encore entamé le jeu de la supplémentarité et qui du même coup ne se soit pas encore laissée entamer par lui [...]. (Derrida, 1967a, p. 344)

A life that has not yet broached the play of supplementarity and which at the same time has not yet let itself be violated by it [...]. (Derrida, 1976b, p. 242)

Dans le premier cas, Spivak marque la distinction annoncée dans sa préface entre broach et breach. Dans le deuxième, alors qu'on aurait pu s'attendre à lire «at the same time has not yet itself be breached by it», Spivak utilise plutôt le verbe violate, qui se présente à cette seule occasion pour traduire entame. On peut sans doute y voir une simple erreur - en l'occurrence, la réédition de 2016 de la traduction corrige ce passage ${ }^{10}$. Si Spivak a bien vu l'importance de l'indécidable entame, notamment par l'évocation qu'en fait Derrida dans Positions,

10. Désormais, le passage se lit: "A life that has not yet broached the play of supplementarity and which at the same time has not yet let itself be breached by it [...]» (Derrida, 2016, p. 263), comme je l'ai proposé (Lemieux, 2015b, p. 284). 
sa traduction ne respecte toutefois pas sa propre règle, à savoir l'usage de broach ou de breach. Cependant, en notant l'importance du concept d'entame dans sa préface, Spivak a contribué à le faire voir sous un nouveau jour. En effet, même si Alan Bass, traducteur entre autres de Marges, utilise peu broach et breach dans ses traductions (alors qu'il devait connaître la préface de Spivak), d'autres traducteurs et traductrices y ont recours. Dans sa traduction de La dissémination (1972), par exemple, Barbara Johnson utilise à de nombreuses reprises les deux formes proposées par Spivak, et ce, conjointement (voir notamment Derrida, 1981, p. 26 et p. 223). Bien que Johnson n'en fasse pas mention, on peut penser que c'est de Spivak quelle prend la double expression, qu'elle a favorisée par rapport au rendu incision de la traduction de Bass de Positions, qui venait tout juste d'être publiée par la même maison d'édition.

Dans la réception qui suivit la traduction de De la grammatologie, au moins un traducteur mentionne Spivak dans sa préface, mais explique du même coup les raisons pour lesquelles il ne croit pas nécessaire de traduire entame par broach ou breach. John P. Leavey Jr., qui a notamment co-traduit Glas (1974), s'explique à ce sujet dans une note intrapaginale sur l'usage du terme freudien Babnung, que Derrida a traduit par frayage dans sa préface à L'Archéologie du frivole : lire Condillac:

As translation and development of Freud's Bahnung, frayage has been variously translated. [David B.] Allison prefers the Standard Edition's "facilitation". [Jeffrey] Mehlman, in the initial translation of "Freud et la scène de l'écriture" for Yale French Studies, introduces "fraying", since this word "captures the violence of the movement of this rudimentary form of writing or "inscription"' (p. 73 of YFS No. 48). [Alan] Bass, in his revised translation of this text (completed before the publication of Spivak's translation of Of Grammatology, but not published until two years later in Writing and Difference), uses "breaching" to render the term. I have used "trailblazing" in the current text for the single occurrence of this term in order to recall the development of Derrida's previous comments on la voie frayée, the marked out trail. Bass, in his use of "breaching", creates a(n) (un)fortunate possibility of confusing frayage and entamer (first cutting, beginning), since Spivak uses "breach" or "broach" to render entamer. In the Husserlian texts by Derrida, Allison and I use "impair" or "undermine" to translate Derrida's seemingly not yet specific use of entamer. (Derrida, 1980, p. 18)

Leavey Jr., qui avait traduit l'«Introduction à L'Origine de la géométrie de Husserl»(Derrida, 1961), montre ici la difficulté qui se 
pose au traducteur lorsqu'il doit juger si la sédimentation conceptuelle à l'œuvre dans la réception s'applique ou non au terme qu'il tente de traduire. Alors que Leavey Jr. traduit, avec Richard Rand, entame par broach dans L'archéologie du frivole et par broach et breach dans Glas (1986), il a préféré, dit-il, ne pas reconnaître le caractère spécifiquement conceptuel du terme dans les textes «husserliens» de Derrida, donc dans le genre, pourrait-on penser, des textes derridiens qui traitent plus précisément de Husserl. Ce qu'exprime ici Leavey Jr., c'est qu'il ne juge pas que Derrida a suffisamment conceptualisé le terme entame pour qu'il mérite une attention particulière dans un glossaire.

Ce jugement vient en quelque sorte compliquer mon hypothèse selon laquelle plus les concepts d'un auteur sont reconnus, plus les traducteurs ont tendance à traduire selon la Lettre. Le livre La voix et le phénomène (1967), le plus «husserlien» des livres de Derrida et le seul à avoir été traduit deux fois, me permettra de nuancer cette hypothèse, à savoir, dans le cas présent, que la retraduction permettra d'uniformiser la terminologie reconnue par la communauté des traducteurs. Le tableau 2 fournit un aperçu des traductions d'entame en anglais ${ }^{11}$.

Tableau 2. Traduction d'entame dans les versions anglaises de La voix et le phénomène

\begin{tabular}{|l|l|l|}
\cline { 2 - 3 } \multicolumn{1}{l|}{} & $\begin{array}{l}\text { Speech and Phenomena } \\
\text { (trad. David Allison, } \\
\text { 1973) }\end{array}$ & $\begin{array}{l}\text { Voice and Phenomenon } \\
\text { (trad. Leonard Lawlor, } \\
\text { 2010) }\end{array}$ \\
\begin{tabular}{|l} 
Fonction verbale, forme \\
affirmative (4)
\end{tabular} & $\begin{array}{l}\text { cut off, enter, strikes, } \\
\text { impair }\end{array}$ & $\begin{array}{l}\text { undermine (2), open } \\
\text { up, cuts }\end{array}$ \\
$\begin{array}{l}\text { Fonction verbale, forme } \\
\text { négative (3) }\end{array}$ & $\begin{array}{l}\text { does not diminish, does } \\
\text { not impair (2) }\end{array}$ & $\begin{array}{l}\text { does not cut, does not } \\
\text { undermine, does not } \\
\text { impair }\end{array}$ \\
$\begin{array}{l}\text { Fonction adjectivale, } \\
\text { forme négative (3) }\end{array}$ & $\begin{array}{l}\text { not affected, not } \\
\text { impaired (2) }\end{array}$ & not impaired (3) \\
$\begin{array}{l}\text { Fonction adjectivale } \\
\text { ("inentamé») (1) }\end{array}$ & unshaken & unmarred \\
\hline
\end{tabular}

11. Pour une vue d'ensemble des occurrences en contexte telles qu'elles se présentent dans les traductions de La voix et le phénomène, on pourra consulter l'Annexe B. 
On remarque immédiatement (tableau 2) la non-cohérence des rendus du terme entame et de ses dérivés, tant dans la première traduction que dans la seconde. Ainsi, 7 verbes sont employés pour rendre les 11 occurrences d'entamer dans la version de David Allison : cut off, enter, strike, impair, diminish, affect et unshake, le plus courant étant impair avec 5 occurrences ( $\approx 64 \%)$. On répertorie 5 verbes différents dans la version de Leonard Lawlor, soit undermine, open up, cut, impair et unmar, le plus courant étant encore une fois impair (4 occurrences, $\approx 36 \%$ des cas). Si on ne peut certes pas parler d'uniformisation du lexique derridien, on ne peut pas non plus parler d'homogénéisation à l'intérieur du texte. Alors que dans le cas d'Allison, on peut légitimement affirmer qu'il y a non-reconnaissance de l'indécidable - spécialiste de Husserl, Allison traduit le livre de Derrida en fonction du champ des études husserliennes aux États-Unis -, on ne peut pas supposer la même chose de Lawlor, qui était sans doute au courant du problème relatif à la traduction d'entame, dont Spivak, rappelonsle, pose les bases dès 1976. Il faut alors se tourner vers le propos de Leavey Jr. : si Lawlor n'accorde aucun privilège au terme entame, jusqu'à ne pas le mentionner dans sa préface, cela signifie qu'il ne pense pas sa traduction en fonction d'un champ d'études dominé par une certaine conception de l'auteur Derrida. Il est vrai que Lawlor a été l'étudiant d'Allison, à qui il dédit d'ailleurs sa traduction. Un tour d'horizon de ses publications nous montre que Lawlor, professeur de philosophie à la Pennsylvania State University, se spécialise dans la philosophie esthétique et la philosophie continentale. Ses travaux portent sur Edmund Husserl, mais aussi sur Martin Heidegger, Maurice MerleauPonty (dont il a traduit les notes de cours de 1955-1956), Paul Ricœur, Gilles Deleuze, Michel Foucault et Renaud Barbaras (qu'il a aussi traduit). La seule autre traduction de Derrida qu'il a effectuée est le texte que celui-ci a rédigé après la mort de Deleuze (Derrida, 1998; repris dans Derrida, 2001).

Cette non-homogénéité de la terminologie en dit peut-être moins sur l'état de la réception de la pensée de Derrida en Amérique que sur celui de la réception de la pensée de Husserl. Si la traduction de Lawlor a bien quelques aspects d'une traduction tardive dans la réception d'un auteur $^{12}$, elle semble quand même prendre l'œuvre de Derrida pour ce quelle énonce et moins pour qui l'a énoncé. Le centre d'intérêt demeure

12. Chose somme toute rare chez les traducteurs et traductrices contemporains de Derrida, Lawlor donne à l'intérieur de chevrons simples la pagination de l'œuvre originale. Il s'agit là d'un signe d'un début de canonisation du texte original. 
Husserl, et Derrida, comme cétait le cas dès sa première traduction américaine en 1973, fait ici figure de commentateur d'Husserl.

On peut ainsi conclure que la dernière traduction de La voix et le phénomène ne respecte pas l'hypothèse selon laquelle la retraduction a tendance à uniformiser les termes. Elle montre toutefois que la réception de Derrida est multiple: la retraduction de La voix et le phénomène s'inscrit dans une tradition philosophique assez éloignée de celle des lecteurs et spécialistes plus traditionnels de Derrida, qui se situent généralement dans le champ des études littéraires, comme le décrivent Michèle Lamont (1987) et François Cusset (2013). Avec cette autre réception, on continue à traduire le quoi plutôt que le qui; mais ce que révèle ce phénomène, c'est que Derrida est pris comme un commentateur encore utile pour comprendre Husserl. Derrida n'est pas traduit pour qui il est, mais pour ce qu'il apporte (encore) dans ce champ d'études.

\section{Conclusion : de la traduction d'un auteur à l'étude de sa réception}

Lorsque Berman posait comme principe qu'une retraduction apporterait un souci plus grand des traducteurs pour la Lettre, il n'avait pas tort, mais il faut en quelque sorte renverser ses présupposés : ce n'est pas l'ceuvre qui demande des retraductions; ce sont les retraductions qui ont pour effet la constitution de l'euvre. L'institution de la retraduction, à la fois comme continuation de la traduction et comme traduction à nouveau d'un même texte, ne peut avoir lieu que si la traduction passe elle-même par une "traduction", cette fois du quoi au qui. Ce travail métatraductif s'analyse en partie en regardant ce qui se passe dans la réception de l'œuvre.

Dans le cas présenté ici, si on voulait faire un parallèle bibliométrique des citations en français et en anglais des premières œuvres de Derrida, on se rendrait rapidement compte que, dans le monde francophone, La voix et le phénomène n'a jamais été mis à part des autres œuvres de la même époque comme De la grammatologie ou L'écriture et la différence. En anglais, une distinction bibliométrique s'effectue, ce que prouvent la traduction et la retraduction. Dans le monde anglophone, La voix et le phénomène est un livre à part, réservé à des spécialistes de la phénoménologie de Husserl, ce qui montre aussi que la phénoménologie, hétérogène au champ des études derridiennes plus reconnues et reconnaissables (la fameuse "Yale School»), est encore très forte aux États-Unis. Si Derrida est généralement vu aux États-Unis comme un «poststructuraliste», on ne dira jamais qu'il 
est postphénoménologue ou postphénoménologiste - et pour cause, ce courant qui n'existe pas n'a pas lieu d'être : lire Husserl est encore possible, bien que cela demeure une activité relativement peu répandue comparée à la lecture de Derrida, par exemple. Il ne s'agit donc pas de conclure qu'un groupe utilise Derrida plus qu'un autre : si une telle délimitation existe entre des factions, chacune s'organise par l'usage qu'elle fait du texte, pour ses concepts ou pour son auteur, et chacune en retire un certain capital symbolique qu'elle peut échanger dans ses propres réseaux.

Étudier la distribution et la vitesse de diffusion des traductions d'un concept philosophique permet, en définitive, de mieux comprendre comment s'élabore la recontextualisation d'une œuvre dans sa réception. Si l'étude de la traduction des concepts de sciences humaines et sociales doit être prise en compte, c'est parce qu'elle permet non seulement de comprendre la réception d'un auteur, mais en outre d'analyser la mécanique de la reconnaissance d'un concept philosophique dans la communauté des lecteurs. Il s'agissait moins ici d'expliciter une histoire de la réception phénoménologique de Derrida aux États-Unis que de marquer l'importance, dans une épistémologie plus générale en l'histoire des idées, de la co-organisation de la traduction et de la réception, et ainsi participer à cette "géopolitique intellectuelle» annoncée par Louis Pinto (2002) : pour comprendre le phénomène de retraduction de l'œuvre, il faut prendre en compte sa réception, tout comme pour comprendre l'histoire mondiale de la circulation des idées, il faut parfois se plonger dans la plus petite unité traduite, même celle qui nous semble la plus insignifiante.

\section{Références}

Bensimon, Paul (1990). «Présentation du numéro "Retraduire"». Palimpsestes, 4, p. ix-xiii.

Berman, Antoine (1990). «La retraduction comme espace de la traduction». Palimpsestes, 4, p. 1-7.

Berman, Antoine (1995). Pour une critique des traductions: John Donne. Paris, Gallimard.

Berman, Antoine (1999). La traduction et la lettre, ou L'auberge du lointain. Paris, Seuil.

Brownlie, Siobhan (2002). «La traduction de la terminologie philosophique». Meta, 47, 3, p. 296-310.

Cassin, Barbara (2004). «Dasein ». In B. Cassin, dir. Le Vocabulaire européen des philosophies. Dictionnaire des intraduisibles. Paris, Seuil/Le Robert.

Chouraqui, André, trad. (1985). La Bible. Paris, Desclée de Brouwer.

Cusset, François (2013). French Theory: Foucault, Derrida, Deleuze et Cie et les 
mutations de la vie intellectuelle aux États-Unis. Paris, La Découverte.

Derrida, Jacques (1961). «Introduction à L'Origine de la géométrie de Husserl». In $\mathrm{E}$. Husserl, L'Origine de la géométrie, trad. Jacques Derrida. Paris, Presses Universitaires de France.

Derrida, Jacques (1967a). De la grammatologie. Paris, Éditions de Minuit.

Derrida, Jacques (1967b). La voix et le phénomène: introduction au problème du signe dans la phénoménologie de Husserl. Paris, Presses Universitaires de France.

Derrida, Jacques (1967c). L'écriture et la différence. Paris, Seuil.

Derrida, Jacques (1972a). La dissémination. Paris, Seuil.

Derrida, Jacques (1972b). Marges de la philosophie. Paris, Éditions de Minuit.

Derrida, Jacques (1972c). Positions : entretiens avec Henri Ronse, Julia Kristeva, Jean-Louis Houdebine, Guy Scarpetta. Paris, Éditions de Minuit.

Derrida, Jacques (1972d). "Positions: Interview with Jacques Derrida conducted by J.-L. Houdebine and Guy Scarpetta». Diacritics, 2, 4, p. 35-43.

Derrida, Jacques (1973). Speech and Phenomena, and Other Essays on Husserl's Theory of Signs. Trad. David Allison, Evanston, Northwestern University Press.

Derrida, Jacques (1974). Glas. Paris, Galilée.

Derrida, Jacques (1976a). L'Archéologie du frivole. Lire Condillac. Paris, Denoël/ Gonthier.

Derrida, Jacques (1976b). Of Grammatology. Trad. Gayatri Chakravorty Spivak. Baltimore, Johns Hopkins University Press.

Derrida, Jacques (1980). Archeology of the Frivolous. Reading Condillac. Trad. John P. Leavey Jr. Pittsburgh, Duquesne University Press.

Derrida, Jacques (1981). Dissemination. Trad. Barbara Johnson. Chicago, University of Chicago Press.

Derrida, Jacques (1986). Glas. Trad.John P. Leavey Jr. et Richard Rand. Lincoln, University of Nebraska Press.

Derrida, Jacques (1987). «Des tours de Babel». In Psyché. Inventions de l'autre. Paris, Galilée.

Derrida, Jacques (1990). Le problème de la genèse dans la philosophie de Husserl. Paris, Presses Universitaires de France.

Derrida, Jacques (1998). «I'm Going to Have to Wander All Alone». Trad. Leonard Lawlor. Philosophy Today, 42, 1, p. 3-5.

Derrida, Jacques (2001). The Work of Mourning. Trad. Pascale-Anne Brault et Michael Naas. Chicago, University of Chicago Press.

Derrida, Jacques (2010). Voice and Phenomenon. Introduction to the Problem of the Sign in Husserl's Phenomenology. Trad. Leonard Lawlor. Evanston, Northwestern University Press.

Derrida, Jacques (2016). Of Grammatology. Trad. Gayatri Chakravorty Spivak. $40^{\text {th }}$ Anniversary Edition. Baltimore, Johns Hopkins University Press.

Heim, Michael H. et Andrzej W. Tymowski (2006). «Recommandations pour la traduction des textes des sciences humaines». Trad. Bruno Poncharal. 
New York, American Council of Learned Societies. [https://www.acls.org/ uploadedFiles/Publications/Programs/sstp_guide_french.pdf].

Ladmiral, Jean-René (1981). «Éléments de traduction philosophique ». Langue française, 51, p. 19-34.

Lamont, Michèle (1987). «How to Become a Dominant French Philosopher: The Case of Jacques Derrida». American Journal of Sociology, XVIII, 3, p. 584-622.

Lemaistre de Sacy, Louis-Isaac, trad. (1759). La Bible de Sacy.

Lemieux, René (2009). «Force et signification à l'épreuve de la traduction : la différance derridienne et son transport à l'étranger». Recherches sémiotiques/ Semiotic Inquiry, 29, 2-3, p. 33-58.

Lemieux, René (2015a). «Retour de Babel : l'indécidabilité derridienne et la rétrotraduction en supplément». Punctum, 1, 2, p. 68-80.

Lemieux, René (2015b). L'Im-possible: Américanité de Jacques Derrida. Une critique sémiopolitique de la traductibilité d'un auteur. Thèse de doctorat. Montréal, Université du Québec à Montréal. Inédit. [http://www.archipel. uqam.ca/8400/].

Littré, Émile (1874). Dictionnaire de la langue française. Tome deuxième $D-H$. Paris, Librairie Hachette.

Lucy, Niall (2004). A Derrida Dictionary. Londres, Blackwell.

Pinto, Louis (2002). "(Re)traductions. Phénoménologie et "philosophie allemande" dans les années 1930». Actes de la recherche en sciences sociales, 145, p. 21-33.

Ramond, Charles (2001). Le vocabulaire de Jacques Derrida. Paris, Ellipses.

Reiß, Katharina (1976). Texttyp und Übersetzungsmethode. Der operative Text. [Type de texte et méthode de traduction. Le texte opérationnel]. Kronberg im Taunus, Scriptor.

Rey, Alain (2006). Dictionnaire historique de la langue française. Paris, Le Robert. Santiago, Silviano (1976). Glossário de Derrida. Rio de Janeiro, Francisco Alves. Spinoza, Baruch (2006). Abrégé de grammaire hébraïque. Trad. Joël Askénazi et Jocelyne Askénazi-Gerson. $3^{\mathrm{e}}$ éd. Paris, Librairie philosophique J. Vrin.

Spivak, Gayatri Chakravorty (1976). «Translator's Preface». In J. Derrida, Of Grammatology, trad. Gayatri Chakravorty Spivak. Baltimore, Johns Hopkins University Press, p. ix-xc.

Wallerstein, Immanuel (1981). «Concepts in the Social Sciences: Problems of Translation». In M. Gaddis Rose, ed. Translation Spectrum. Essays in Theory and Practice. Albany, State University of New York Press, p. 88-98.

René Lemieux

Département des arts, langues et littératures

Université de Sherbrooke Sherbrooke (Québec), Canada rene.lemieux@usherbrooke.ca 


\section{Annexe A \\ Le terme entame et ses dérivés dans De la grammatologie}

\section{En français (1967)}

[...] question qui entame la philosophie [...] et se laisse recouvrir par elle [...] (p. 34)

[...] la particularité de l'exemple n’entame pas la généralité de notre propos [...] (p. 44)

[...] irruption du dehors dans le dedans, entamant l'intériorité de l'âme [...] (p. 52)

Mais ces critiques n'entament pas [...] l'intention profonde de Saussure $[\ldots]$ (p. 65; note 8$)$

$[\ldots]$ cet index et quelques autres $[\ldots]$ nous donnent déjà le moyen assuré d'entamer la dé-construction [...] (p. 68)

Ce qui entame le mouvement de la signification, c'est ce qui en rend l'interruption impossible. (p. 72)

Elle était ce qui, au plus proche, menaçait le désir de la parole vive, ce qui du dedans et dès son commencement, l'entamait. (p. 83)

[...] cet équilibre se confond avec cela même qui entame la linéarité du symbole. (p. 127)

[...] ce qui, mesuré à la taille de l'écriture, s'entame seulement dans l'epistémè. (p. 142)

Aucune intégrité n'a été entamée. (p. 166)

\section{En anglais (1976)}

[...] a question that broaches philosophy [...] and lets itself be taken over by philosophy [...] (p. 20-21)

[...] the particularity of the example does not interfere with the generality of my argument [...] (p. 29)

[...] eruption of the outside within the inside, breaching into the interiority of the soul [...] (p.34)

But these criticisms do not interfere [...] with Saussure's profound intention [...] (p. 326; note 8)

[...] this and some other indices [...] already give us the assured means of broaching the de-construction [...] (p. 46)

What broaches the movement of signification is what makes its interruption impossible. (p. 49)

It threatened the desire for the living speech from the closest proximity, it breached living speech from within and from the very beginning. (p. 5657)

[...] this balance is confused with the very thing that broaches the linearity of the symbol. (p. 85)

[...] measured against the shape of writing, it is broached only in the epistémè. (p. 93)

No integrity has been breached. (p. 113) 
[...] il prête l'oreille et entame une première complicité [...] (p. 167)

Elle a commencé par entamer l'aliénation [...] (p. 206)

<suite> [...] et elle finit par laisser entamée la réappropriation. (p. 206)

Le supplément rend fou parce qu'il [...] entame dès lors et notre plaisir et notre virginité. (p. 222)

[...] une certaine déconstruction pourrait être entamée de cette totalité [...] (p. 231)

[...] le procès indéfini de la supplémentarité a toujours déjà entamé la présence [...] (p. 233)

On doit [...] l'aimer à une distance suffisante pour que les forces $[. .$.$] n'en$ soient pas entamées. (p. 252)

Elle entame l'histoire. (p. 259)

[...] une étrange différence qui les constitue en les entamant [...] (p. 283)

[...] l'invasion des Barbares septentrionaux entame un nouveau cycle de dégénérescence historique [...] (p. 288-289)

[...] chaque nouveau cycle entame une progression-régression [...] (p. 289)

[...] au lieu de conclure de cette simultanéité que le chant s'entamait dans la grammaire [...] (p. 307)
[...] he lends an ear and broaches a first complicity [...] (p. 113)

Differance began by broaching alienation [...] (p. 143)

<suite> [...] and it ends by leaving reappropriation breached. (p. 143)

The supplement is maddening because it $[\ldots]$ consequently breaches both our pleasure and our virginity. (p. 154)

[...] a certain deconstruction of that totality [...] might be broached. (p. 162)

[...] the indefinite process of supplementarity has always already infiltrated presence [...] (p. 163)

One must [...] love her, from a sufficient distance so that the forces [...] are not breached through it. (p. 177)

It broaches history. (p. 182)

[...] a strange difference which constitutes it by breaching it [...] (p. 198)

[...] the invasion of the northern barbarians ushered in a new cycle of historical degeneration. (p. 202)

[...] each new cycle begins a progression-regression [...] (p. 202203)

[...] instead of concluding from this simultaneity that the song broached itself in grammar [...] (p. 215) 
[...] le manque, en s'ajoutant comme un plus à un plus, entame une énergie [...] (p. 308)

Et il l'entame bien comme un supplément dangereux [...] (p. 308)

[...] elle a toujours déjà entamé la présence dans la différance [...] (p. 308)

Avant donc que d'entamer l'histoire de notre espèce $[. .$.$] (p. 316; citation$ de Rousseau, manuscrit L'influence des climats sur la civilisation dans l'édition de La Pléiade : p. 530)

[...] celle-ci comporte une plus grande menace d'absence et entame l'énergie de la vie $[. .$.$] (p. 336)$

Une vie qui n'ait pas encore entamé le jeu de la supplémentarité [...] (p. 334)

<suite> [...] et qui du même coup ne se soit pas encore laissée entamer par lui $[\ldots]$ (p. 344)

Celle-ci entame le langage [...] (p. 344)

[...] mais laissant au négatif et au positif une pureté inentamée. (p. 349)

Ce modèle exemplaire d'un souffle (pneuma) pur et d'une vie inentamée [...] (p. 353)

[...] mais assez pure pour rester inentamée par le travail de la différence $[\ldots]$ (p. 353)
[...] the lack, adding itself as a plus to a plus, cuts into an energy [...] (p. 215)

And indeed it breaks in as a dangerous supplement [...] (p. 215)

[...] it has always already broached presence in difference $[\ldots]$ (p. 215)

Thus before one broaches the history of our species [...] (p. 221; même référence dans la traduction)

$[\ldots]$ the latter is attended by a greater threat of absence and cuts into life's energy. (p. 236)

A life that has not yet broached the play of supplementarity [...] (p. 242)

$<$ suite $>[. .$.$] and which at the same$ time has not yet let itself be violated by it $[\ldots]$ (p. 242)

It broaches language [...] (p. 242)

[...] yet allowing an intact purity to both the negative and the positive. (p. 246)

With this exemplary model of a pure breath (pneuma) and of an intact life [...] (p. 249)

[...] but pure enough to have remained unblemished by the work of difference [...] (p. 249) 
En elle la société s'entame et se differre. (p. 377)

Si la culture s'entame ainsi dans son point d'origine $[. .$.$] (p. 377)$

Dans cette entame, ce qui s'initie s'est déjà altéré [...] (p. 377)

[...] par lequel l'accent s'entame dans l'articulation, se diffère en s'espaçant. (p. 378)

[...] une nouvelle distinction est nécessaire qui entamerait jusqu'au propre de l'expression [...] (p. 391)

La théorie de l'origine sensible des idées en général [...] découpe ici sa critique du rationalisme de type cartésien sur un fond théologique et métaphysique inentamé. (p. 400)

La visibilité [...] est toujours ce qui, la séparant d'elle-même, entame la voix vivante. (p. 432)
In it society is broached and is deferred from itself. (p. 267)

If culture is thus broached within its point of origin [...] (p. 267)

In this broaching, what is initiated is already corrupted [...] (p. 267)

[...] by which accent is broached within articulation, is deffered through spacing. (p. 268)

[...] a new distinction is necessary which would infiltrate as far as the literalness [propre] of expression [...] (p. 276-277)

The theory of the sensible origin of ideas in general [...] here exhibits its Cartesian critique of rationalism against an intact theological and metaphysical base. (p. 282)

Visibility [...] is always that which, separating it from itself, breaches [entame] the living voice. (p. 306) 


\section{Annexe B \\ Le terme entame et ses dérivés dans La voix et le phénomène}

\author{
En français (1967) \\ Cela $[. .$.$] n'entame pas la valeur$ \\ fondatrice de la présence. (p. 5)
}

Or, puisque la conscience transcendantale n'est pas entamée dans son sens [...] (p. 12)
Première version en anglais (1973) et deuxième version en anglais (2010)

This [...] nor does it diminish the founding value of presence. (p. 7) It does not cut into the founding value of presence. (p. 6)

But since the meaning of the transcendental consciousness is not affected [...] (p. 13)

Now, since transcendental consciousness is not impaired in its sense $[\ldots]$ (p. 11)

[...] cette nécessité factuelle [...] ne doit pas [...] entamer la possibilité d'une rigoureuse distinction d'essence. (p. 21)

[...] this de facto necessity [...] must not $[\ldots]$ cut off the possibility of a rigorous distinction of essence. (p. 20) $[\ldots]$ this factual necessity [...] must not $[\ldots]$ undermine the possibility of a rigorous essential distinction. (p. 18)

[...] il faut traquer la pureté inentamée de l'expression. (p. 22)

[...] je ne peux pas entamer un discours «effectif» [...] (p. 56)

Cette unité n'entame pas la distinction d'essence [...] (p. 63) $[\ldots]$ we have to ferret out the unshaken purity of expression [...] (p. 22)

$[\ldots]$ it is necessary to track down the unmarred purity of expression. (p. 19)

[...] I cannot enter into an "effective" discourse [...] (p. 50)

[...] I cannot open up an "actual" discourse [...] (p. 43)

This unity does not impair the essential distinction [...] (p. 56)

This unity does not undermine the essential distinction [...] (p. 48) 
Cette intimité $[. .$.$] entame en sa$ racine l'argument de l'inutilité du signe $[\ldots]$ (p. 74)
The fact $[\ldots]$ strikes at the very root of the argument for the uselessness of signs $[\ldots]$ (p. 66)

This intimacy [...] cuts into, at its root, the argument for the uselessness of the sign [...] (p. 57)

[...] un médium qui n'entame pas la présence et la présence à soi des actes [...] (p. 85)

[...] a medium that does not impair the presence and self-presence of the acts $[\ldots]$ (p. 76)
[...] a medium that does not impair the presence and self-presence of the acts $[\ldots]$ (p. 65)

Il est impliqué structurellement dans mon opération [...] que son unité ne soit pas entamée par l'absence de perception bic et nunc. (p. 103)

\section{[...] si elle ne se laisse pas entamer} par la fausseté [...] (p. 108)
It is structurally implied in my performance $[. .$.$] that its unity is$ not impaired by the absence of perception here and now. (p. 92-93)

What is implied structurally in what I am doing is [...] that its unity is not impaired by the absence of the hic et nunc perception. (p. 79)

$[\ldots]$ if it is not impaired by falsity [...] (p. 96)

[...] if it does not let itself be impaired by falsehood [...] (p. 82)

[...] the theory which claims it would [...] impair its ideality [...] (p. 100) [...] the theory according to which the fluctuation $[. .$.$] would thus$ undermine its ideality [...] (p. 86) 